\title{
MINIMAL AND MAXIMAL PRODUCT DIFFERENTIATION IN HOTELLING'S DUOPOLY
}

\author{
Nicholas ECONOMIDES \\ Columbia University, New York, NY 10027, USA
}

Received 27 June 1985

Final version received 13 January 1986

Hotelling's (1929) model of duopolistic competition is re-examined. A family of utility functions is used which has as a special case Hotelling's original utility function. In a two-stage location-price game it is shown that an equilibrium exists when the curvature of the utility functions in the space of characteristics is sufficiently high. The (subgame-perfect) equilibrium never exhibits minimum product differentiation. On the other hand, not all equilibria are at maximal product differentiation.

\section{Introduction and statement of the problem}

Hotelling (1929) claimed that competition in differentiated products results in minimal differentiation. D'Aspremont et al. (1979) showed that, because a key calculation in Hotelling's model is incorrect, the minimum differentiation equilibrium does not exist. Proposing a similar utility function they exhibited a maximal differentiation equilibrium. Here we analyse the problem for a class of utility functions and assess the generality of the maximum differentiation result.

In his acclaimed study 'Stability in Competition', Hotelling (1929) considered the following paradigm of duopolistic competition for differentiated products. Two firms are selling products 1 and 2 at prices $p_{1}$ and $p_{2}$ respectively. Products 1 and 2 are identical in all respects except for one characteristic. Let $x$ be the amount of characteristic embodied in product 1 and $y$ in product 2 . Consumers are endowed with utility functions separable in money (Hicksian composite commodity) and utility derived from a differentiated product. Each consumer is constrained to buy one unit of one of the differentiated products. Consumers have single peaked preferences in the space of potential differentiated products. Consumers are distributed uniformly in $[0,1]$ according to the product they prefer most.

Competition takes place in two stages. In the last-stage (short-run) game products are fixed and firms compete in prices. The first-stage (long-run) game is defined for product configurations such that a unique Nash equilibrium in prices exists in the last-stage game. In the first-stage game firms compete non-cooperatively in product specifications with instantaneous adjustment to the Nash equilibrium prices. The utility of consumer $z$ who has money $m$ and buys product $x$ at price $p_{1}$ is: $U_{z}\left(m, p_{1}, x\right)=m-p_{1}+k-f(d(x, z))$, where $k$ is the utility of product ' $z$ ' to consumer ' $z$ ' (assumed to be a large constant), $d(x, z)$ is the (Euclidean) distance in the product space, and $f(d)$ is an increasing function of $d$ passing through the origin. ${ }^{1}$ Hotelling (1929) took $f(d)$ to be linear.

1 There is a formal equivalence of this model with a model where products are delivered at the factory door and consumers bear the costs of transportation that are given by function $f(d)$. 
Then:

(1a) For $x \neq y$ existence of a unique Nash equilibrium in prices in the last-stage game is restricted to regions such that $x$ and $y$ are sufficiently far apart [d'Aspremont et al. (1979)]. For a fixed location $y$ of the opponent, the locations $x<y$ which result in a Nash equilibrium in the price subgame lie in an interval $\left[0, x_{\mathrm{e}}(y)\right)$, with $x_{\mathrm{e}}(y)<y$. Equilibrium prices are strictly positive.

(1b) For $x=y$ Bertrand's equilibrium occurs at zero prices.

(2) An equilibrium in the location game does not exist because marginal relocations in the directions of the best replies bring the firms into the region where the Nash equilibrium in the (last-stage) price game does not exist.

D'Aspremont et al. (1979) showed that a utility function quadratic in distance $\left[f(d)=d^{2}\right]$ results in the existence of price equilibria in the last-stage game for any locations, and that in the long run there exist subgame-perfect equilibria at maximal product differentiation. Thus, at first glance it seems that the existence of price equilibria is intimately connected with maximal differentiation in the location game. We explore this problem using a family of utility functions. A natural family of utility functions is the one implied by the family of transportation cost functions $f(d)=d^{\alpha}$, $1 \leqslant \alpha \leqslant 2$

Let $E(\alpha)$ be the set of pairs of locations (products) $(x, y)$ such that the non-cooperative price subgame played for these locations results in a unique equilibrium. Let the realized profits for each firm at this equilibrium be $\pi_{1}^{v}(x, y), \pi_{2}^{v}(x, y)$. We define the direciion in which $\mathrm{d} \pi_{1}^{v} / \mathrm{d} x$ is positive as the 'relocation tendency' of firm 1, and similarly for firm 2. A (subgame-perfect) equilibrium of the location-price game must be at the zero relocation locus, $\mathrm{d} \pi_{1}^{\prime \prime} / \mathrm{d} x=\mathrm{d} \pi_{2}^{\prime \prime} / \mathrm{d} y=0$. We establish the zero relocation locus for all $\alpha$. Its intersection with the existence region $E(\alpha)$ defines equilibrium of the price-location game.

\section{Relocation tendencies in the long-run game}

The utility of consumer $z$ that buys product $x$ at price $p_{1}$ is $U_{z}(m, x)-m+k-p_{1}-|x-z|^{\alpha}$. Utility maximization is equivalent to picking the minimum of $p_{1}+|x-z|^{\alpha}$ and $p_{2}+|y-z|^{\alpha}$. Let $\bar{z}\left(p_{1}, p_{2}\right)$ be the solution in $z$ of

$p_{1}+|x-z|^{\alpha}=p_{2}+|z-y|^{\alpha}$.

Consumer $\bar{z}$ is indifferent between buying from firm 1 and 2 . All consumers in $[0, \bar{z}$ ) buy from firm 1 and all consumers in $(\bar{z}, 1]$ buy from firm $2 .^{2}$

Demand functions are $D_{1}\left(p_{1}, p_{2}\right)=\bar{z}\left(p_{1}, p_{2}\right)$ and $D_{2}\left(p_{1}, p_{2}\right)=1-\bar{z}\left(p_{1}, p_{2}\right)$. Profits are $\pi_{1}=$ $p_{1} D_{1}, \pi_{2}-p_{2} D_{2}$. The first-order conditions for profit maximization are ${ }^{3}$

$p_{1}^{\mathrm{m}}=\alpha \bar{z}\left[(\bar{z}-x)^{\alpha-1}+(y-\bar{z})^{\alpha-1}\right], \quad p_{2}^{\mathrm{m}}=\alpha(1-\bar{z})\left[(\bar{z}-x)^{\alpha-1}+(y-\bar{z})^{\alpha-1}\right]$.

\footnotetext{
2 Implicitly it has been assumed that $\bar{z}$ lies between $x$ and $y$. It turns out that this is implied by the existence of equilibrium in the price game.

3 Second-order local conditions are also satisfied.
} 


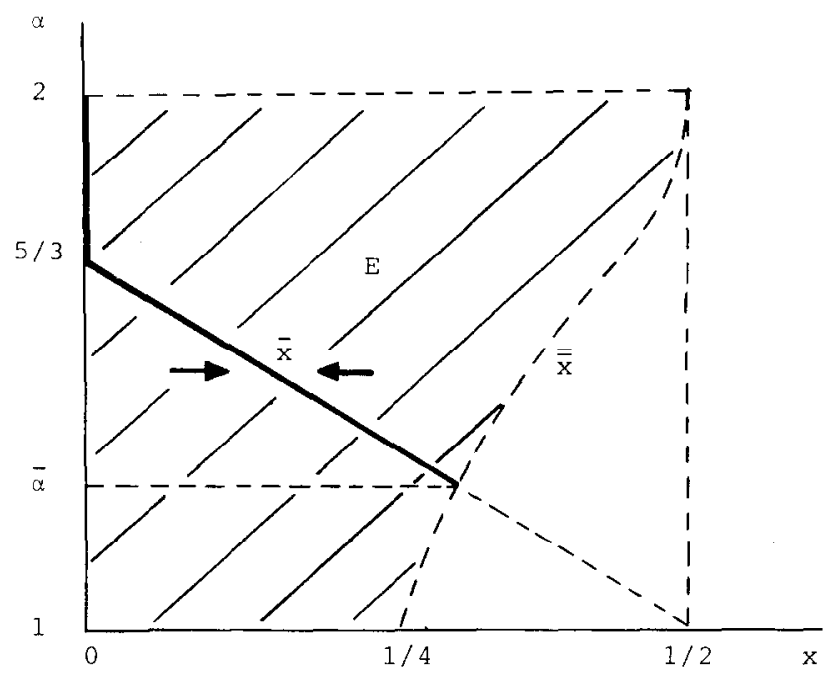

Fig. 1. The existence region $E$ and the locus of equilibium $\bar{x}$.

Eliminating $p_{1}$ and $p_{2}$ between (1) and (2) results in

$g(\bar{z}, x, y) \equiv \alpha\left[(\bar{z}-x)^{\alpha-1}+(y-\bar{z})^{\alpha-1}\right](2 \bar{z}-1)+(\bar{z}-x)^{\alpha}-(y-\bar{z})^{\alpha}=0$.

The equality $g(\bar{z}, x, y)=0$ holds for all $\bar{z}$ that correspond to the Nash equilibrium prices $\left(p_{1}^{*}, p_{2}^{*}\right)$, which are the solution of eqs. (2). The corresponding equilibrium profits are $\pi_{1}^{v}(x, y)=\pi_{1}\left(p_{1}^{*}(x, y)\right.$, $\left.p_{2}^{*}(x, y)\right)=\alpha \bar{z}^{2}\left[(\bar{z}-x)^{\alpha-1}+(y-\bar{z})^{\alpha-1}\right] . \pi_{1}^{\prime \prime}(x, y)$ [and correspondingly $\pi_{2}^{v}(x, y)$ ] are the objective functions of the game in varieties (locations).

As mentioned above, the relocation tendency for firm 1 follows the sign of the derivative of the equilibrium profits $\pi_{1}^{b}$ with respect to $x$. Thus it follows the direction of the best reply of firm 1 to the location choice of firm 2. The effect on profits of changes in $x$, keeping $y$ constant (evaluated for symmetric locations, $y=1-x)$ is $\mathrm{d} \pi_{1}^{v} / \mathrm{d} x=k(2-4 x-3(\alpha-1))$, for $x \neq 1 / 2$ where $k$ is a positive constant. ${ }^{4}$ Therefore $\mathrm{d} \pi_{1}^{i} / \mathrm{d} x \gtreqless 0 \Leftrightarrow x \lesseqgtr 5 / 4-3 \alpha / 4$. Let $\bar{x}(\alpha)=5 / 4-3 \alpha / 4$. Then starting at any $x$ firm 1 will tend to move toward $\bar{x}$. We have established:

Proposition 1. In the location game a symmetric equilibrium exists and is given by $\bar{x}(\alpha)=5 / 4-3 \alpha / 4$, $\bar{y}(\alpha)=1-\bar{x}(\alpha)$ for $\alpha$ in $[1,5 / 3]$ and by $\bar{x}(\alpha)=0, \bar{y}(\alpha)=1$ for $\alpha$ in $(5 / 3,2]$, provided that the corresponding price subgame has an equilibrium.

Fig. 1 shows the locus $\bar{x}$ of zero relocation tendencies.

${ }^{4} \mathrm{~d} \pi_{1}^{v}(x) / \mathrm{d} x=\partial \pi_{1}^{v} / \partial x+\left(\mathrm{d} \pi_{1}^{v}(\bar{c}) / \mathrm{d} \bar{z}\right) \cdot(\mathrm{d} \bar{z} / \mathrm{d} x) \cdot \partial \pi_{1}^{v} / \partial x=-\alpha(\alpha-1) \bar{z}^{2}(\bar{z}-x)^{\alpha-2}<0 . \mathrm{d} \pi_{1}^{v}(\bar{c}) / \mathrm{d} \bar{z}=2 \alpha \bar{z}\left[(\bar{z}-x)^{\alpha-1}+\right.$ $\left.(y-\bar{z})^{\alpha-1}\right]+\alpha(\alpha-1) \bar{z}^{2}\left[(\bar{z}-x)^{\alpha-2}-(y-\bar{z})^{\alpha-2}\right]$. Let $A=\left[(\bar{z}-x)^{\alpha-1}+(y-\bar{z})^{\alpha-1}\right]$ and $B=\left[(\bar{z}-x)^{\alpha-2}-(y-\right.$ $\left.\bar{z})^{\alpha-2}\right]$. Then $\mathrm{d} \pi_{1}^{\prime \prime}(\bar{c}) / \mathrm{d} \bar{z}=\alpha \bar{z}[2 A+(\alpha-1) \bar{z} B] . \partial g / \partial x=-\alpha(\alpha-1)(\bar{z}-x)^{\alpha-2}(2 \bar{z}-1)-\alpha(\bar{z}-x)^{\alpha-1}, \partial g / \partial \bar{z}=3 \alpha A+$ $\alpha(\alpha-1) B(2 \bar{z}-1)$, so that $\mathrm{d} \bar{z} / \mathrm{d} x=\left[(\alpha-1)(\bar{z}-x)^{\alpha-2}(2 \bar{z}-1)+(\bar{z}-x)^{\alpha-1}\right] /[3 A+(\alpha-1) B(2 \bar{z}-1)]$. Therefore, $\mathrm{d} \pi_{1}^{v} / \mathrm{d} x$ $\stackrel{=\alpha(\alpha}{=} 1) \bar{z}^{2}(\bar{z} \quad x)^{\alpha-2}+\alpha \bar{z}[2 A+(\alpha-1) B \bar{z}]\left[(\alpha-1)(\bar{z}-x)^{\alpha-2}(2 \bar{z}-1)+(\bar{z}-x)^{\alpha-1}\right] /[3 A+(\alpha-1) B(2 \bar{z}-1)]$. For symmetric $x$ and $y, x+y=1, \bar{z}=1 / 2, A=2(1 / 2-x)^{\alpha-1}, B=0$, and therefore $\mathrm{d} \pi_{1}^{o} / \mathrm{d} x=\alpha(1 / 2-x)^{\alpha-2}[5-4 x-$ $3 \alpha)] / 12$. 


\section{Regions of existence of equilibrium in the price subgame}

In the original model of Hotelling with linear utility in the characteristics space $(\alpha=1)$ the demand and profit functions are discontinuous. However, it is not the discontinuity of the payoff function which creates the non-existence of an equilibrium. It is the failure of the quasi-concavity of the profit function, resulting in discontinuous best reply functions which creates the non-existence problem. For $\alpha \neq 1$ continuity is restored hut the quasi-concavity still fails for all $\alpha<1 .^{5}$

In the linear case, price undercutting forces directly the non-existence of equilibrium because it results in the opponent having zero profits, while each firm (if $x \neq y$ ) can guarantee a positive amount of profits by quoting a sufficiently low price. For a non-linear utility function, optimal undercutting may not result in the opponent firm making zero profits.

In general the profit functions in this problem are double-peaked. ${ }^{6}$ We call the lower of the two prices associated with the peaks the optimal undercutting price. Formally we define the optimal undercutting price as $p_{1}^{\mathrm{u}}\left(p_{2}\right)$ which fulfills: $p_{1}^{\mathrm{u}}+(\bar{z}-x)^{\alpha}=p_{2}+(\bar{z}-y)^{\alpha}$ and $\bar{z} \leqslant 1$. If the $\bar{z}$ which solves the above system is infeasible, then we define as the optimal undercutting price for firm 1 the $p_{1}^{\mathrm{u}^{\prime}}\left(p_{2}\right)$ which fulfills $p_{1}^{\mathrm{u}^{\prime}}+(1-x)^{\alpha}=p_{2}+(1-y)^{\alpha}$. While $p_{1}^{\mathrm{u}}$ leaves some positive market share to the opponent, $p_{1}^{\mathrm{u}^{\prime}}$ leaves zero market share to him.

We first examine the case where optimal undercutting drives the opponent out of business. ${ }^{7}$ Equilibrium profits are $\pi_{1}^{*}=p_{1}^{*} / 2=\alpha(1 / 2-x)^{\alpha-1} / 2$, while undercutting profits are $\pi_{1}^{\mathrm{u}}=p_{1}^{\mathrm{u}}=$ $\alpha(1 / 2-x)^{\alpha-1}+x^{\alpha}-(1-x)^{\alpha}$. Let the difference of the above functions be $f(x, \alpha)=\pi_{1}^{\mathrm{u}}-\pi_{1}^{*}=$ $\alpha(1 / 2-x)^{\alpha-1} / 2+x^{\alpha}-(1-x)^{\alpha}$. When $f \leqslant 0$, undercutting is unprofitable and an equilibrium exists. Since $f(1 / 2, \alpha)=0$ and $\partial f(1 / 2, \alpha) / \partial x<0,{ }^{8} f(\cdot)$ must be positive for some $x<1 / 2$. Since also ${ }^{9} f(0, \alpha)<0$, there exists a solution $\overline{\bar{x}}$ in $(0,1 / 2)$ to the equation $f(x)=0$. Since the third derivative of $f(\cdot)$ is globally negative, ${ }^{10}$ the second derivative is decreasing and can only change sign once. This establishes the uniqueness of $\overline{\bar{x}}$, because more than one solution of $f(x)=0$, together with the fact that $f(0)<0$ and $f(1 / 2-\epsilon)>0$ imply more than one solution of $\partial^{2} f(x) / \partial x^{2}=0$, which, as mentioned above, is not true. Thus, an equilibrium exists for all $x$ (and corresponding $y=1-x$ ) in $[0, \overline{\bar{x}})$. The analysis of the case when optimal undercutting does not drive the opponent out of business (and $p_{1}^{\mathrm{u}}$ is used) follows similarly through numerical techniques. ${ }^{11}$ The boundary of the overall existence region is $\overline{\bar{x}} \simeq 1.40-2.76 \alpha+2.08 \alpha^{2}-0.46 \alpha^{3} . \overline{\bar{x}}(\alpha)$ is increasing in $\alpha$. In the linear case of Hotelling (1929), where $\alpha=1$, we have $\overline{\bar{x}}=1 / 4$, while in the extreme quadratic case $(\alpha=2)$ we have $\overline{\bar{x}}=1 / 2$. The locus of $\overline{\bar{x}}$ is seen in fig. 1 .

The shaded region of existence of equilibrium in the price subgame, $E(\alpha, x)$, seen in fig. 1 , contains a part of the locus of zero relocation tendencies $\bar{x}(\alpha)$. These locations in $E$, together with their counterparts $\bar{y}(\alpha)=1-\bar{x}(\alpha)$ constitute equilibria of the location game which are subgame perfect in the price subgame. Let $\bar{\alpha}$ be the intersection of $\bar{x}(\alpha)$ and $\overline{\bar{x}}(\alpha)$. Location equilibria exist in the interior of the product space for $\alpha$ in $(\bar{\alpha}, 5 / 3)$ on $\bar{x}(\alpha), \bar{y}(\alpha)=1-\bar{x}(\alpha)$. Corner equilibria exist for $\alpha$ in $[5 / 3,1]$ at $x=0, y=1$. For $\alpha<\bar{\alpha}$ no locational equilibria exist. Hotelling's (1929) case falls in this last category.

5 See Economides (1984) for a detailed study of the duopoly of Hotelling (1929). There the non-existence problem is fixed through the introduction of not too high reservation prices.

6 This double-peak property is shown by plotting these functions using a dense grid of values of prices.

7 Optimal undercutting makes the market share of the opponent zero if $\partial \pi_{1}\left(p_{1}=p_{1}^{\mathbf{u}^{\prime}}\right) / \partial p_{1}<0$ which is equivalent to $\alpha(1 / 2-x)^{\alpha-1}+x^{\alpha}-(1-x)^{\alpha}-\alpha(1-x)^{\alpha-1}+\alpha x^{\alpha-1}>0$.

$8 \partial f / \partial x=\alpha\left[-(\alpha-1)(1 / 2-x)^{\alpha-2} / 2+x^{\alpha-1}+(1-x)^{\alpha-1}\right]$, and $\lim _{x \rightarrow 1 / 2} \partial f / \partial x=-\infty$, for $\alpha<2$.

$f(0, \alpha)=\alpha / 2^{\alpha}-1<0$.

$10 \partial^{2} f / \partial x^{2}=\alpha(\alpha-1)\left[(\alpha-2)(1 / 2-x)^{\alpha-3} / 2+x^{\alpha-2}-(1-x)^{\alpha-2}\right] . \quad \partial^{3} f / \partial x^{3}=\alpha(\alpha-1)(\alpha-2)\left[-(\alpha-3)(1 / 2-x)^{\alpha-4} / 2+\right.$ $\left.x^{a-3}+(1-x)^{a-3}\right]$. Clearly, the expression in the brackets is positive which makes the third derivative globally negative.

11 The computer routines used are available from the author. 
Proposition 2. Subgame-perfect equilibria exist in the location-price duopoly game a-la-Hotelling for transportation cost functions $f(d)=d^{\alpha}$ with $2 \geqslant a>\bar{\alpha} \simeq 1.26$. For $\bar{\alpha}>\alpha \geqslant 5 / 3$ the locations of the equilibria are strictly interior to $[0,1]$, while for $\alpha \geqslant 5 / 3$ the location of the equilibrium is at the corners of $[0,1]$. For $\alpha<\bar{\alpha}$ there are no equilibria.

\section{Conclusion}

We have therefore established two results: First, the principle of minimum differentiation does not hold in duopoly price-location games a-la-Hotelling, since there is no long-run subgame-perfect equilibrium with minimum product differentiation. Second, the opposing principle of maximum differentiation is also incorrect in general. Although maximal product differentiation holds for very convex 'transportation cost' functions (large $\alpha$ 's), there exists a range of utility functions (parameterized by intermediate $\alpha$ 's) such that the perfect equilibrium locations are interior points of the product space.

\section{References}

D'Aspremont, C., J. Jaskold Gabszewicz, J.F. Thisse, 1979, On Hotelling's 'Stability in competition', Econometrica 47, 1145-1150.

Economides, N., 1984, The principle of minimum differentiation revisited, European Economic Review 24, $345-368$.

Hotelling, H., 1929, Stability in competition, The Economic Journal 39, 41-57.

Lancaster, K., 1979, Variety, equity and efficiency (Columbia University Press, New York).

Novshek, W., 1980, Equilibrium in simple spatial (or differentiated product) models, Journal of Economic Theory 22 , 313-326.

Salop, S., 1979, Monopolistic competition with outside goods, Bell Journal of Economics 10, 141-156.

Selten, R., 1975, A reexamination of the perfectness concept in extensive game forms, International Journal of Game Theory 4, 25-55. 